\title{
BRIEF REPORT \\ Primitive Neuroectodermal Tumor (PNET) of the Uterus in a Renal Allograft Patient: A Case Report
}

\author{
Edward Peres, MD, ${ }^{1 *}$ Tej K. Mattoo, $\mathrm{MD}^{2}{ }^{2}$ Janet Poulik, $\mathrm{MD}^{3}$ and Indira Warrier, $\mathrm{MD}^{1}$
}

\begin{tabular}{|c|c|}
\hline $\begin{array}{l}\text { The incidence of malignancy after renal } \\
\text { transplant has been reported to range from } 4 \% \\
\text { to } 18 \% \text {. Tumors of the skin and lip tend to be the } \\
\text { most common with non-Hodgkin lymphoma } \\
\text { comprising } 20 \% \text { of all neoplasms. Primitive } \\
\text { neuroectodermal tumors (PNET) are collectively } \\
\text { described as being a part of the Ewing sarcoma } \\
\text { family of tumors. PNET occur more commonly } \\
\text { in the second decade of life, predominantly } \\
\text { affecting Whites and Hispanics, and rarely } \\
\text { occur in individuals of African or Asian descent. }\end{array}$ & $\begin{array}{l}\text { The most common primary site of involvement } \\
\text { is along the central axis, particularly the chest } \\
\text { (Askin tumor), but it can arise in any soft tissue. } \\
\text { PNET also occur in the head and neck. PNET } \\
\text { involving the cervix, urinary bladder, uterus, } \\
\text { and vagina have been reported. We describe a } \\
\text { case of a 15-year-old female who, } 9 \text { years after } \\
\text { receiving a living related renal transplant, } \\
\text { developed a post-transplant PNET of the uterus. } \\
\text { Pediatr Blood Cancer 2005;44:283-285. } \\
\text { () } 2004 \text { Wiley-Liss, Inc. }\end{array}$ \\
\hline \multicolumn{2}{|c|}{ Key words: cancer biology; Ewings/PNET; transplantation } \\
\hline
\end{tabular}

\section{INTRODUCTION}

Although renal transplantation has dramatically changed the outcome of end stage renal disease, it is associated with significant side effects including opportunistic infections, graft rejection, drug toxicity, and malignancy [1]. The incidence of malignancy after renal transplant has been reported to range from $4 \%$ to $18 \%$ and may result from pre-existing cancers in recipients, donortransmitted cancers, or de novo malignancies [2,3]. Other predisposing factors for the development of malignancies include immunosuppressive agents, carcinogenic effects of medications, activation of oncogenic viruses, uremia, and antigenic stimulation from the transplant $[3,4]$. Tumors of the skin and lip tend to be the most common, while non-Hodgkin lymphoma comprises $20 \%$ of all neoplasms [3,5]. In this report, we describe a 15year-old female who, 9 years after receiving a living related renal transplant, developed a post-transplant primitive neuroectodermal tumor (PNET) of the uterus.

\section{CLINICAL HISTORY}

A 15-year-old Asian American female with a history of a renal allograft presented with a 2-day history of emesis, loose stools, suprapubic abdominal pain, and fever up to $101.3^{\circ} \mathrm{F}$. She had also complained of increased vaginal bleeding for the past 4 months. There was no history of weight loss, hematuria, or dyspnea though she did complain of urinary frequency and urgency without dysuria. She had received a living related donor renal transplant 9 years previously for end stage renal disease, secondary to membranoproliferative glomerulonephritis type I. The post-transplant immunosuppressive therapy included cyclosporin, Imuran, and corticosteroids. Three episodes of acute rejection were treated with brief courses of high dose corticosteroids and one course of OKT3. On physical examination her temperature was $38.5^{\circ} \mathrm{C}$, heart rate was 130, and her blood pressure was normal. Abdominal examination revealed a $6 \mathrm{~cm} \times 7 \mathrm{~cm}$ suprapubic mass, which was firm, fixed, and tender. There was no evidence of lymphadenopathy or hepatosplenomegaly.

Urinalysis revealed 50-100 WBC, $2+$ protein, and was positive for leukocyte esterase and nitrates. Both urine and blood cultures grew Escherichia coli and intravenous antibiotic therapy was started empirically. Investigation of the suprapubic mass by ultrasonography and magnetic radiographic imaging (MRI), revealed a large intrauterine cystic mass (Figs. 1 and 2). The mass extended into and through the cervical os, with involvement of bilateral iliac nodes, suggestive of a uterine malignancy. At laparotomy, a necrotic mass within the uterus was noted and she underwent a gross total resection of the tumor including hysterectomy with lymph node biopsy. Post-operative imaging revealed no evidence of metastatic disease.

\footnotetext{
${ }^{1}$ Department of Pediatric Hematology/Oncology, Children's Hospital of Michigan and Wayne State University School of Medicine, Detroit, Michigan

${ }^{2}$ Department of Nephrology, Children's Hospital of Michigan and Wayne State University School of Medicine, Detroit, Michigan

${ }^{3}$ Department of Pathology, Children's Hospital of Michigan and Wayne State University School of Medicine, Detroit, Michigan

*Correspondence to: Dr. Edward Peres, Division of Hematology/ Oncology, Children's Hospital of Michigan 3901 Beaubien Blvd. Detroit, Michigan 48201. E-mail: eperes@dmc.org
}

Received 11 May 2004; Accepted 7 September 2004 


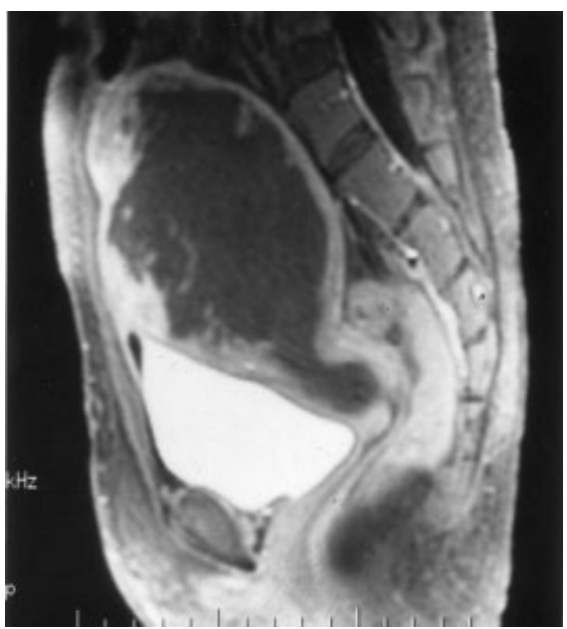

Fig. 1. Magnetic radiographic imaging of the pelvis revealing a large cystic mass in the uterine cavity with myometrial invasion and extension into and through the external cervical os.

Microscopic examination revealed the uterine wall to be infiltrated by large nests of undifferentiated small round blue cells. Tumor cells had coarsely granular to stippled chromatin with a high nuclear to cytoplasmic ratio and numerous mitotic figures throughout (Figs. 3 and 4). Immunohistochemical stains revealed the tumor cells to be intensely immunoreactive for MIC2 protein (CD 99), a marker for PNET/EWING tumor that identifies glycoprotein p30/32, vimentin (a soft tissue marker), and cytokeratin reactivity (a marker for epithelial tissue). The neoplastic cells were positive for PAS, negative for pancytokeratin. The tumor showed focal strong positivity with, synaptophysin (a marker for neurons) and neuron specific enolase (a marker for nerve tissue), all markers consistent with PNET. Cytogenetics revealed a der(6). RTPCR amplification of mRNA extracted from the tumor failed to demonstrate the EWS-FLI 1 translocation and

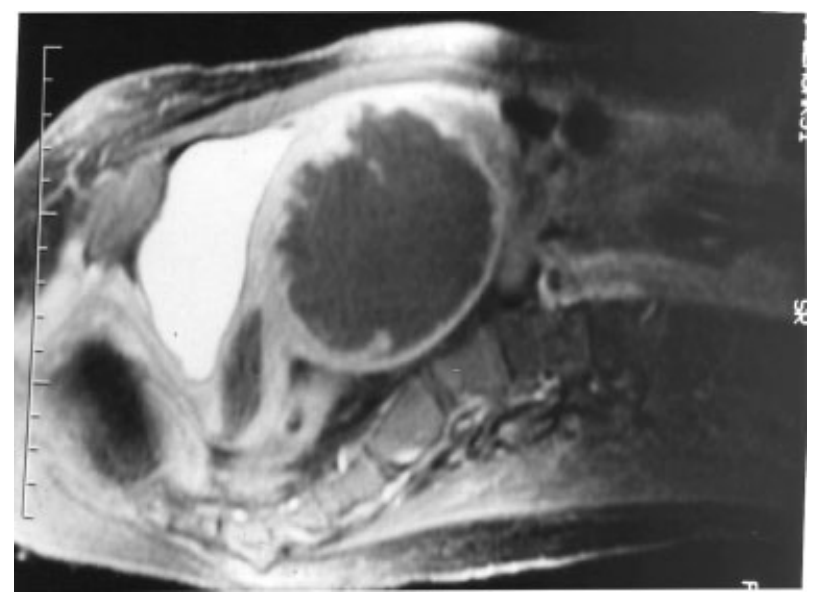

Fig. 2. Magnetic radiographic imaging of the pelvis revealing a large cystic mass in the uterine cavity with myometrial invasion and extension into and through the external cervical os.

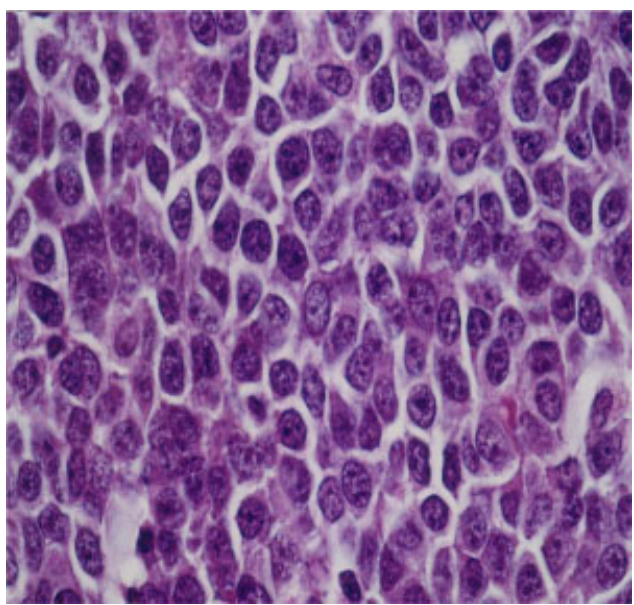

Fig. 3. Photomicrograph showing uniform round cells with moderately and small-sized nuclei. (H \& E 400×). [Color figure can be viewed in the online issue, which is available at www.interscience.wiley.com.]

subsequent analysis for the $E R G$ gene on chromosome 21 and the ETV-1 gene on chromosome 7 were also negative. However, a small percentage of EWS/PNET fail to show a $\mathrm{t}(11 ; 22)$ and $\operatorname{der}(6)$ has been reported in a subset of PNET cases.

She received the first course of chemotherapy consisting of carboplatin $\left(450 \mathrm{mg} / \mathrm{m}^{2}\right)$ and etoposide $\left(200 \mathrm{mg} / \mathrm{m}^{2}\right)$. This was complicated by deterioration in renal function. The second course of chemotherapy was complicated by severe renal, liver, and respiratory failure and pancytopenia, which responded to aggressive therapy. Three weeks later, her renal function returned to baseline with a serum creatine of $1.5 \mathrm{mg} / \mathrm{dl}$. Repeat radiological imaging revealed no evidence of tumor. In view of the significant toxicity she experienced during her first two courses of

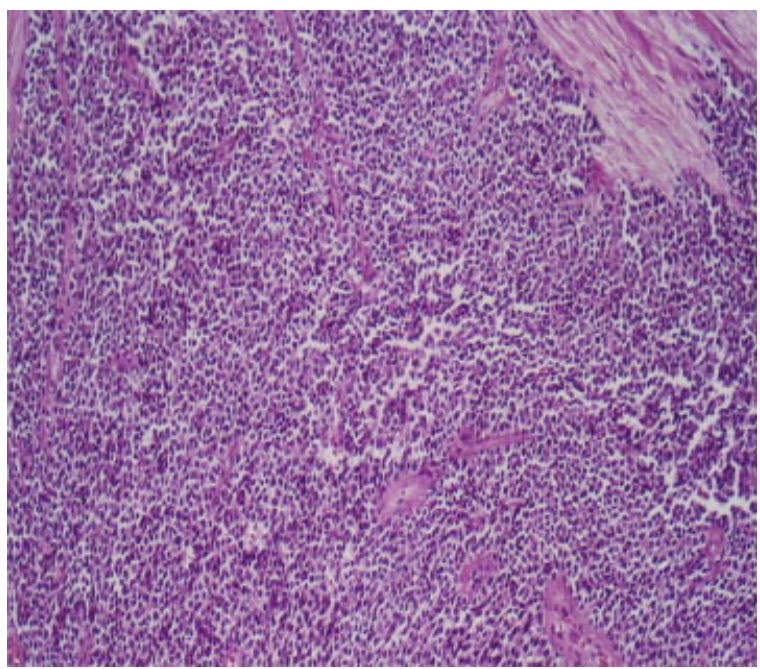

Fig. 4. Photomicrograph showing the uterine wall infiltrated by large nest of undifferentiated round cells. (H \& E 100×). [Color figure can be viewed in the online issue, which is available at www.interscience. wiley.com.] 
chemotherapy, her parents refused all additional therapy. At follow-up 12 months from her last chemotherapy treatment, she has stable renal function (BUN $32 \mathrm{mg} / \mathrm{dl}$; creatine $2.2 \mathrm{mg} / \mathrm{dl}$ ) and has no radiographic evidence of tumor recurrence.

\section{DISCUSSION}

PNET was first described by Arthur Purdy Stout in the early part of this century [7]. It is a highly malignant tumor and microscopically described as a small round cell tumor focally arranged as rosettes [10]. PNET are collectively described as being a part of the Ewing sarcoma family of tumors $[7,8]$. PNET occurs more commonly in the second decade of life, predominantly affecting Whites and Hispanics and rarely occurring in individuals of African or Asian descent $[7,8]$. The most common primary site of involvement is along the central axis, particularly the chest (Askin tumor), but it can arise in any soft tissue [8,9]. PNET also occur in the head and neck. PNET involving the cervix, urinary bladder, and vagina have also been reported [10].

The differential diagnoses of small round cell tumor in our patient included uterine lymphoma, small cell carcinoma, and PNET/Ewing sarcoma. Post-transplant PNET of the uterus has not been reported and is rare in patients of Asian decent. Our patient's tumor had extension and invasion into the cervical os and invasion of the uterine wall, findings, which were suggestive of a more aggressive tumor. PNET tend to have a more aggressive biological behavior with invasion and infiltration into the surrounding tissue with distant metastases. At diagnosis as many as $25 \%$ of patients have detectable metastatic disease to lung, bone, and bone marrow; and nearly all patients have micrometastases [10,11]. Our patient did not have any detectable metastatic disease at the time of diagnosis and her lymph node biopsy was negative for disease. Although our patient's tumor failed to demonstrate the $\mathrm{t}(11 ; 22)(\mathrm{q} 24 ; \mathrm{q} 12)$ (EWS-FLI 1) translocation by RT-PCR, which is detectable in $85 \%$ of PNET cases, cytogenetics revealed a der(6), which has been previously reported; immunohistochemistry and microscopic evaluation was consistent with PNET [7].

According to the Cincinnati Transplant Registry (CTTR), data on 11,359 post-transplant related malignancies have occurred in 10,667 organ allograft recipients with no reports of post-transplant uterine PNET reported as of 1998 [13]. The predominant tumors are lymphomas, carcinomas of the skin, lips, vulva, perineum, and in situ carcinoma [14]. Excluding Kaposi sarcoma, the incidence of sarcoma was $1.8 \%$ with only one case of Ewing sarcoma being reported [13]. PNET is a rare tumor comprising $1 \%$ of all sarcomas; it is extremely rare in people of Asian origin and has not been reported to occur in post-transplant recipients [5,13]. The predisposing conditions leading to the development of PNET is unclear, although immunosuppression might have been a contributing factor.

\section{REFERENCES}

1. Ranadive NU, Urmi C, Kumar M. Primary primitive neuroectodermal tumor of the kidney: A case report. Arch Esp Urol 1999;52: 190-192.

2. Tripathy LN, Forster DMC, Timperley WR. Adult primitive neuroectodermal tumor. A case report and review of the literature. Brit J Neurosurg 1999;13:90-92.

3. Niaude P. Posttransplant lymphoproliferative disease following renal transplantation: A multicenter retrospective study of 41 cases observed between 1992 and 1996. Transplant Proceed 1998;30: 2816-2817.

4. Desai RA, Desai SB, Amonkar PP, et al. Primary primitive neuroectodermal tumor of the parotid gland. Histopathology 1998;33: 375-378.

5. de Alava E, Gerald WL. Molecular biology of the Ewing's sarcoma/primitive neuroectodermal tumor family. J Clin Oncol 2000;18:204-213

6. Deleted in proofs.

7. Horowitz ME, Malawer MM, Woo SY, et al. Ewing's Sarcoma family of tumors: Ewing's Sarcoma of bone and soft tissue and the peripheral primitive neuroectodermal tumors. In: Pizzo PA, Poplack DG, editors. Principles and practice of pediatric oncology. 3rd edition. Philadelphia: Lippincott Raven Publ.; 1997. pp $831-$ 863.

8. Angervall L, Enzinger FM. Extraskeletal neoplasm resembling Ewing's sarcoma. Cancer 1975;36:205-240.

9. Dehner L. Primitive neuroectodermal tumor and Ewing's sarcoma. Am J Surg Pathol 1993;17:1-13.

10. Zucman J, Delattre O, Desmaze C, et al. Cloning and characterization of the Ewing's sarcoma and peripheral neuroepithelioma $\mathrm{t}(11 ; 22)$ translocation breakpoints. Genes Chromos Canc 1992;5: $271-277$.

11. Jurgens H, Bier V, Harms D, et al. Malignant peripheral neuroectodermal tumors. Cancer 1988;61:349.

12. Enzinger FM, Weiss SW. Primitive neuroectodermal tumors and related lesions. In: Enzinger FM, Weiss SW, editors. Soft tissue tumors. 3rd edition. St. Louis: CV Mosby Co.; 1995. pp 929-964.

13. Penn I. Sarcomas in organ allograft recipients. Transplantation 1995;60:1485-1491.

14. Penn I. Post-transplant malignancy: The role of immunosuppression. Drug Saf 2000;23:101-113. 